Relmecs, diciembre 2018 - mayo 2019, vol. 8, no. 2, e046, ISSN 1853-7863

Universidad Nacional de La Plata. Facultad de Humanidades y Ciencias de la Educación.

Centro Interdisciplinario de Metodología de las Ciencias Sociales.

Red Latinoamericana de Metodología de las Ciencias Sociales

\title{
Uso de cuestionarios en investigaciones sobre universidad: análisis de experiencias desde una perspectiva situada
}

Using questionnaires in research on university: analysis of experiences with a situaded perspective

Maria Isabel Pozzo

Instituto Rosario de Investigaciones en Ciencias de la Educación-CONICET, Argentina

pozzo@irice-conicet.gov.ar

Ana Borgobello

Instituto Rosario de Investigaciones en Ciencias de la Educación-CONICET, Argentina

María Paula Pierella

Instituto Rosario de Investigaciones en Ciencias de la Educación-CONICET, Argentina

\section{Resumen:}

El artículo refiere al uso de cuestionarios en investigaciones sobre la universidad, enfatizando una perspectiva metodológica crítica de problemáticas concretas. Luego de un abordaje conceptual, se analizan cuatro estudios de equipos y temas disímiles en los que se utilizó esta técnica. Ellos refieren al uso del cuestionario: en una investigación internacional, una cuasiexperimental, frente a un tema sensible e implementado por Internet. Las conclusiones aluden a la ambigüedad terminológica-metodológica, la importancia de leer los resultados en contexto concebidos en enfoques complejos y situados y el lugar del sesgo en la elaboración del instrumento y el análisis de datos.

Palabras Clave: Universidad, Investigación, Metodología, Cuestionario, Estudios comparados.

\section{ABSTRACT:}

The article refers to the use of questionnaires in research focused on University level from a critical methodological perspective on specific issues. After discussing main concepts, four different studies as regards teams and topics which involve the use of this technique are analyzed. They refer to the use of a questionnaire: in an international research, in a quasi-experimental study, in a sensitive topic, and implemented by the Internet. The main conclusions refer to the ambiguity of concepts and methodological approaches, the importance of reading data in the production context and the bias in the survey design and the data analysis.

KEYWORDS: University, Research, Methodology, Questionnaires, Comparative studies.

\section{INTRODUCCIÓN}

La recolección de información en la investigación constituye un momento fundamental para la producción de nuevos conocimientos. En el campo educativo, entrevistas y observaciones, en sus diversas variantes, gozan de un lugar preponderante frente a las encuestas. La reflexión acerca de la utilización de unas y otras remite a una discusión más amplia que, aunque resuelta en gran medida (Schmelkes, 2001), parece resurgir en algunas circunstancias; concretamente, aquella que tiende a oponer diseños flexibles versus estructurados, respectivamente.

En la trayectoria profesional de las autoras en el ámbito de las humanidades en universidades de Rosario, Argentina, se han encontrado resistencias por parte de la comunidad académica en relación al uso de cuestionarios en investigaciones educativas. Si bien la problemática podría considerarse local, esta situación puede darse también en otros contextos. En la medida en que suele hacerse una aplicación poco fundamentada y escasamente reflexiva de este tipo de instrumento, cabe señalar que dicho rechazo no carece de racionalidad, justificando el debate.

El presente artículo pretende poner en discusión dichas controversias desde una postura crítica, examinando modos en los que se utilizan cuestionarios en investigaciones en educación. En este sentido, se

\section{Recibido: 11 agosto 2017 | Aceptado: 26 octubre 2017| Publicado: 20 diciembre 2018}

Cita sugerida: Pozzo, M. I., Borgobello, A. y Pierella, M. P. (2018). Uso de cuestionarios en investigaciones sobre universidad: análisis de experiencias desde una perspectiva situada. Revista Latinoamericana de Metodología de las Ciencias Sociales, 8(2), e046. https://doi.org/10.24215/18537863e046 
consideraron ventajas y desventajas del cuestionario en la investigación en el ámbito universitario desde una perspectiva metodológica en problemáticas y ejemplos concretos. En los estudios presentados el eje ha sido el ajuste ecológico al objeto de investigación y no la matematización del cuestionario en sentido de medirlo o testearlo. Esto llevó a contextualizar sucintamente cada estudio en el marco en el que fue desarrollado.

Las diferencias organizacionales entre universidades y perfiles docentes hacen que el rasgo institucional más sobresaliente sea la heterogeneidad. Frente a esta situación, desde el punto de vista de la investigación, resulta complejo pensar en estudios que no sean situados.

Es así que el uso de los cuestionarios permite una aproximación amplia a esta diversidad de contextos y realidades en la que se torna necesario reflexionar sobre esta dimensión al encarar estudios que vayan más allá del análisis de un caso. Teniendo en cuenta estos recaudos, el alcance que brindan constituye una herramienta valiosa. Entre otras ventajas, permiten obtener información de sujetos que pertenecen a tribus (Becher, 2001) distantes unas de otras, tanto en términos disciplinares como institucionales.

Desde una perspectiva latinoamericana, la tendencia al uso de cuestionarios suele centrarse en instrumentos construidos en otras latitudes y luego traducidos y validados en el contexto local. Esta última característica se destaca prioritariamente en estudios de psicología educativa al estudiar, entre otros temas, estilos de aprendizaje (Cala Aiello y Riera García, 2014; Ventura y Moscoloni, 2015; Falicoff, Odetti y Domínguez Castiñeiras, 2014), prácticas pedagógicas (Pérez et al., 2016) o burnout en la profesión docente (Bustamante, Bustamante, González y Bustamante, 2016). Este tipo de trabajos muestra como rasgo predominante una preocupación por la matematización del análisis de datos focalizando, por ejemplo, en la confiabilidad y análisis de su estructura factorial (Acosta, 2012; Medrano, Moretti, Ortiz y Pereno, 2013; Noriega Biggio, Vázquez y García, 2015). En un sentido diferente y en menor medida, una serie de trabajos utilizan cuestionarios ad hoc, siendo complementados en algunos casos con otros instrumentos cualitativos como entrevistas en profundidad (Manchini, Penhos y Suárez, 2016).

Por otra parte, es menester situar el tratamiento del tema en un contexto en el que la virtualidad y los medios digitales se inmiscuyen en casi todo el quehacer humano, y por ende, en el de la investigación. Aquí también se adopta una postura crítica acerca del grado de utilidad de los procedimientos automatizados que proporcionan los programas para la implementación y procesamiento de encuestas. $\mathrm{Al}$ respecto, se ha reparado en las limitaciones de herramientas que a simple vista podrían resultar completamente satisfactorias, cobrando esta reflexión un lugar destacado en la literatura sobre enfoque cualitativo.

El desarrollo de secciones comienza por una revisión teórico-metodológica que permite esclarecer conceptos utilizados a menudo indistintamente pero que ameritan su diferenciación. Luego, continúa con cuatro estudios que involucran el uso de cuestionarios que permiten identificar ventajas y desventajas contextualizadamente. El artículo finaliza con la discusión sobre ventajas y limitaciones de esta técnica para la investigación en el contexto universitario.

\section{El CUESTiOnARio como estrategia metodológica}

Como inicio de esta sección, interesa destacar el uso ambiguo e indiferenciado entre encuesta y cuestionario. Si bien, las formas en las que se alude al tema son diversas, por lo general, encuesta alude al método mientras que cuestionario, al instrumento concreto que se aplica.

Específicamente, para Hernández-Sampieri, Fernández-Collado y Baptista-Lucio (2008) los cuestionarios consisten en una serie de preguntas abiertas y/o cerradas respecto de una o más variables a medir y son probablemente el instrumento más utilizado para recolectar datos. Por su parte, Archenti (2007) refiere a sondeo o encuesta -en el mismo nivel-como un método utilizado para recolectar datos sobre actitudes, creencias u opiniones a través de cuestionarios estandarizados.

Cabe aclarar que, en un sentido restringido, el concepto de estandarización proviene del ámbito de la psicometría, es decir, la estandarización remite a pruebas psicométricas que funcionan en base a baremos 
específicos. En ese sentido, se le aplica al cuestionario cuando, por ejemplo, se determina un coeficiente de confiabilidad. En un sentido amplio y, como se toma en este trabajo, aludiría a la idea de protocolización, es decir, de repetición de aplicación altamente similar en distintos casos.

Retomando la diversidad terminológica, Hernández-Sampieri et al. (2008), señalan que las encuestas de opinión (surveys) son investigaciones no experimentales, transversales o transeccionales descriptivas o correlacionales-causales que, generalmente, utilizan cuestionarios. Más allá de la especificidad del tema tratado, que se desarrolla posteriormente en esta sección, resulta significativo que estos autores (a través del verbo ser) equiparan encuestas a cierto tipo de investigación.

En un sentido más general aún, Canales, Alvarado y Pineda (1994) sostienen que la encuesta consiste en una forma de obtener información de sujetos de estudio proporcionada por ellos mismos sobre opiniones, actitudes, conocimientos o sugerencias. Para estos autores, el cuestionario es un método que utiliza un instrumento o formulario impreso, destinado a obtener información sobre el problema en estudio y que el consultado llena por sí mismo.

En síntesis, el cuestionario es mencionado en los libros de metodología de investigación, aun los más clásicos, como el instrumentopropiamente dicho, como método, como diseño, como tipo de investigación, homologado a o diferenciado de encuesta, sondeo, entrevista estructurada, entre otros, lo que justifica la presente revisión.

En este trabajo, como se dijo, aun atendiendo a la multiplicidad de acepciones conocidas, el cuestionario es concebido como el instrumento que se diseña en el marco de un proyecto de investigación elaborado a partir de objetivos específicos.

Un aspecto que podría considerarse ineludible dada su frecuencia en libros de metodología de investigación refiere a ventajas y desventajas de la aplicación de cuestionarios en la producción de conocimiento científico.

La ventaja de poder construir una base estadística lo convirtió en la herramienta cuantitativa típica dado que permite estimar predicciones con apoyo empírico. Así, para Archenti (2007) el principal atractivo de la encuesta como método reside en su potencial predictivo con un margen de error calculable.

Existen ventajas de los cuestionarios autoadministrados como los que se presentan en este artículo que son señaladas recurrentemente: costo relativamente bajo; capacidad de proporcionar información sobre un amplio número de personas en un tiempo breve; facilidad para obtener, cuantificar, analizar e interpretar los datos; respeto al ritmo individual al contestar; bajos requerimientos de personal; posibilidad de mantener el anonimato de los encuestados y eliminar ciertos sesgos que introducen los encuestadores; entre otras (e.g. Canales et al., 1994; Díaz, 2012; Hernández-Sampieri et al., 2008).

El cuestionario en línea, específicamente, presenta algunas ventajas adicionales: inmediatez en el procesamiento de los datos, reducción del trabajo de campo, posibilidad de introducir elementos audiovisuales que contribuyan a la comprensión y menor coste de la investigación. Asimismo, al no existir encuestador, se reduce la intimidación que puede provocar su presencia, lo que implica una mayor garantía de anonimato que ayudaría a que se expresen respuestas más críticas, menos aceptadas socialmente o más sensibles (Coomber, 1997; Díaz, 2012). Coomber (1997), en un texto clásico sobre el tema, resalta el acceso a partir de esta modalidad a poblaciones difíciles de alcanzar ya sea por razones geográficas como debido a temas ilegales, delicados o sensibles. La ampliación de zonas geográficas facilita, por ejemplo, estudios internacionales y sólo requeriría la traducción de la invitación a participar y del cuestionario.

Por el contrario, distintos autores señalan limitaciones (Canales et al., 1994; Coomber, 1997; Díaz, 2012; Hernández-Sampieri et al., 2008): escasa flexibilidad y dificultad para profundizar en la información obtenida; riesgo de que no llegue a los destinatarios esperados o que no se obtenga respuesta; imposibilidad de aclarar dudas sobre las preguntas; dificultad para obtener una tasa alta de cuestionarios completos. Cabe destacar que aquellos que voluntariamente responden y quienes no lo hacen pueden tener opiniones o características predominantes distintas que pueden afectar la investigación (Coomber, 1997). Específicamente cuando se trata de cuestionarios en línea, se encuentran los errores de cobertura, escaso 
control sobre los respondentes y, por tanto, de las muestras obtenidas. Un problema adicional del medio digital refiere a la sospecha de un mensaje con virus, ante el cual el destinatario ni siquiera atine a responder.

Varias de las ventajas y limitaciones del cuestionario como instrumento de investigación coinciden cuando se aplica en línea o en papel. Sin embargo, la dimensión del soporte resulta un tema relevante desde el punto de vista metodológico en cuanto al número de respuestas, representatividad de los respondentes, posibilidad de reiterar la pregunta, etc.

En cuanto a aspectos regionales, si bien en América Latina se fueron abriendo los caminos necesarios para la implementación de cuestionarios en línea, se cuenta con escasos trabajos en comparación con las publicaciones que utilizan esta técnica en el mundo anglosajón, en el que el acceso mismo a internet fue masivizado con anterioridad. Un dato ilustrativo sobre esta disparidad es que en el mismo año en el que Coomber (1997) informaba acerca de las posibilidades brindadas por Internet para la realización de cuestionarios en línea, en Argentina el Poder Ejecutivo Nacional declaraba de interés el acceso a Internet y se comenzaba a desarrollar un plan estratégico para ello. A pesar de ello, los cuestionarios de este tipo ya forman parte de las estrategias consagradas en la investigación social, e incluso se dispone de numerosos softwares que facilitan las condiciones metodológicas para realizarlos (De-Sena y Lisdero, 2015).

\section{EsTUdios QUE INVOLUCRAN EL USO DE CUESTIONARIOS EN LA UNIVERSIDAD}

En esta sección presentaremos estudios diversos que utilizaron cuestionarios como estrategia metodológica, con el objeto de anclar la discusión en casos concretos. A tal efecto, hemos seleccionado cuatro situaciones paradigmáticas que involucran cuestionarios. La primera de ellas refiere a un cuestionario de satisfacción en un estudio internacional, caracterizado por un espectro geográfico muy amplio, al igual que su alcance temporal, dado que se administra a lo largo de dos años. La segunda situación se aboca a ahondar temas sensibles para los respondentes. La tercera desarrolla un cuestionario implementado en línea, poniendo de manifiesto cuestiones propias del soporte y la última se encuadra en un estudio cuasiexperimental con diversidad de perfiles profesionales participantes.

A los efectos de que las presentaciones resulten comparables, la presentación se estructura en torno a los siguientes ejes: contexto en el que se realiza el estudio, enfoque teórico-metodológico, objetivos del cuestionario dentro del estudio, tipo de soporte (papel y/u online), ventajas, limitaciones y dificultades para la tarea investigativa.

Más allá de la variedad de los estudios, todos tienen en común que los cuestionarios aplicados fueron diseñados ad hoc (vale decir, no estandarizados), enmarcardos en un enfoque complejo-dialéctico (Achilli, 2005) y concebidos e implementados en ámbitos universitarios.

\section{El cuestionario de satisfacción en un estudio internacional}

El primer estudio se enmarca en una investigación internacional tanto en su diseño como en su aplicación. El proyecto denominado "Módulos educativos para teoría y práctica en circuitos eléctricos y electrónicos siguiendo una metodología de enseñanza y aprendizaje basada en la pregunta apoyada en VISIR", dirigido por el Dr. Gustavo Alves en el Instituto Politécnico de Porto, Portugal, se encuentra en desarrollo desde octubre de 2015, previsto para un lapso de dos años y medio. Dicho proyecto está financiado por la Unión Europea a través de su Programa Erasmus+; específicamente, de su instrumento "Proyecto de construcción de capacidades en el campo de la educación superior". Su objetivo consiste en ampliar la implementación del laboratorio remoto Virtual Instrument Systems In Reality (VISIR) -creado en el Instituto de Tecnología de Blekinge, Suecia, por Ingvar Gustavsson- hacia América Latina en la formación de ingenieros eléctricos y electrónicos y la enseñanza de la física en carreras afines. A tal efecto, propone un proceso focalizado en 
algunas instituciones de nivel superior de Argentina y Brasil constituidas como casos, a partir de la experiencia con dicho recurso en cuatro universidades europeas de distintos países (Suecia, Austria, España y Portugal). La implementación paulatina y sucesiva requiere de un monitoreo constante que atienda al seguimiento de la opción didáctica elegida (aludida en su título) en los contextos singulares. La capacitación en cadena del proceso de diseminación requiere la evaluación de las diversas instancias formativas. Para constatar la calidad de las enseñanzas y los aprendizajes se vale de un enfoque mixto cuanti-cualitativo y se prevén distintos instrumentos de recolección de información, así como otras fuentes de evidencia espontánea. Entre los primeros, el proyecto contempla la utilización de cuestionarios de satisfacción, así como otras técnicas tales como las observaciones de las capacitaciones, las entrevistas y los diarios de campo. Entre las segundas, se encuentran los propios módulos educativos y planificaciones de los docentes que incorporarán este recurso a sus clases. Este apartado se aboca a los cuestionarios de satisfacción aplicados al cabo de cada sesión de capacitación. Estos fueron elaborados por el grupo abocado a la recolección de datos, conformado por una de las autoras de este artículo, junto a Elsa Dobboletta y tres investigadoras del Instituto Politécnico de Porto, Portugal: las Dras. Clara Viegas, Arcelina Márquez y la Lic. Natércia Lima.

Los cuestionarios se instrumentaron fundamentalmente en versión papel, por varias razones. Ante todo, para cerciorarse de contar con la participación masiva de los asistentes in situ, antes de retirarse del lugar del evento. Además, porque permite administrar el cuestionario en el momento justo; esto es, apenas terminadas las sesiones, cuando es posible una reflexión retrospectiva, pero a su vez con el recuerdo aún nítido de la experiencia. La versión en línea se utilizó solamente para la modalidad virtual del cursado o para aquellos que por alguna contingencia no estuvieron presentes en el momento de la administración del cuestionario.

El recurso de un instrumento estandarizado permitió recabar información comparable para evaluar implementaciones en contextos diversos: en dos países disímiles como Brasil y Argentina, en instituciones diferentes (privada y pública, universidades e institutos universitarios), en diversos momentos y a cargo de distintos capacitadores. De no haber sido así, el proceso se dispersaría en numerosos registros empíricos difíciles de procesar y aun de transcribir, o bien resultaría incapturable, dificultado por las distancias espaciotemporales. En cambio, permitió concentrar experiencias distantes en el tiempo y en el espacio. El siguiente detalle puede explicar de manera elocuente la amplitud témporo-espacial. La primera implementación del cuestionario fue tras la primera capacitación, que consistió en dos sesiones que por sus características y disertantes disímiles ameritaban ser evaluadas por separado. Así, los 60 cuestionarios recolectados se distribuyen en asistentes presenciales/virtuales, de América Latina/Europa y Sesión 1/2.

La segunda capacitación se desarrolló no ya en un mismo espacio/tiempo, sino en las distintas instituciones latinoamericanas de nivel superior participantes. Tampoco coincidieron temporalmente, si bien se desarrollaron a lo largo de dos meses, lo que le dio uniformidad con respecto a los tiempos del proyecto. La diferencia en cuanto a número de respondentes se debió a la idiosincrasia de cada institución y región. La distribución de los 87 cuestionarios obtenidos en esta nueva implementación se desglosa por país e institución participante.

Otra de las ventajas de los cuestionarios refiere a la posibilidad con la que cuentan los respondentes para expresar matices respecto a su grado de conformidad. A través del abanico de opciones que proporciona una escala likert, se contrarrestan las limitaciones de un instrumento cerrado.

Efectivamente, las respuestas predeterminadas limitan las expresiones a distintos grados de valoración, sin permitir explicar por qué lo perciben de esa forma. Sin embargo, el recurso de una o dos preguntas abiertas al final permite canalizar dichas sutilezas. Depende de la cooperación de los respondentes aprovechar dichas preguntas, y responderlas con el desarrollo que sea conveniente.

Dentro de la estandarización que caracteriza a esta opción metodológica, la construcción requiere atender la naturaleza de las dimensiones evaluadas. Así, por ejemplo, cuando el aspecto implicado es la sensibilidad de los instructores a los intereses y preocupaciones de los participantes de una capacitación, los valores a consignar en el cuestionario fueron: Insatisfactorio, Inferior a la media, Promedio, Superior a la media, 
Excelente. Ahora bien, estas opciones no resultan aplicables para la pregunta que persigue evaluar si las sesiones de capacitación cubrieron las expectativas del respondente. Para ella se propuso: En absoluto, Parcialmente, Satisfactoriamente, Muy satisfactoriamente, Completamente. Otra situación a evaluar refería al grado de dificultad del manejo del recurso. En tal caso, las opciones fueron: Demasiado difícil, Dificil, Normal, Fácil, Demasiado fácil.

Así expuestas estas cuestiones metodológicas pueden resultar muy evidentes y hasta obvias. Sin embargo, el diseño indiferenciado del instrumento, sin ajustar preguntas y respuestas, puede desorientar al respondente, desaprovechando la instancia. Preguntar por la dificultad proponiendo como posibles respuestas: insatisfactorio, inferior a la media, etc., resultaría en una incongruencia que atentaría contra la validez de la información recolectada.

La traducción del cuestionario - especialmente las opciones de las respuestas-constituyó otro desafío. En tal sentido, algunas expresiones como insatisfactorio, excelente o difícil, resultaban transparentes al pasar de una lengua a otra (en este caso, del inglés al portugués y el español). Otras en cambio, planteaban interrogantes en cuanto a frecuencia de uso en cada comunidad lingüística. Así, mientras que average, below average, son frecuentes en el mundo anglosajón, su traducción textual no lo es tanto en el hispanohablante. A los fines de la equiparación de las respuestas, se optó por la traducción literal.

\section{El cuestionario frente a un tema sensible}

El proyecto denominado "Contextualización y análisis de prácticas académicas deshonestas de estudiantes universitarios. Un estudio exploratorio-descriptivo en la Facultad de Psicología de la Universidad Nacional de Rosario (UNR)" se propuso realizar un análisis descriptivo de las prácticas académicas deshonestas que estudiantes de primero, quinto y sexto año manifestaban realizar y observar en sus compañeros en una muestra de 560 estudiantes de la Carrera de Psicología.

Por tanto, el objetivo general del estudio se centró en conocer y analizar a partir de su contexto las narrativas de los estudiantes sobre las prácticas transgresoras en las que incurrían consciente o inconscientemente (copia en examen o plagio, respectivamente) para entender los motivos que los llevan a realizar tales prácticas.

El estudio en su conjunto tuvo un abordaje complejo en el que se utilizó un cuestionario en papel -con preguntas cerradas y abiertas- y luego se solicitaron opiniones acerca de los resultados a estudiantes del último año de la carrera a través de correo electrónico. El cuestionario original era español, elaborado por Sureda-Negre, Comas-Forgas y Gili-Planas (2009) y piloteado y adaptado para su aplicación de acuerdo al léxico corriente en el ámbito académico argentino. Al cuestionario español, que indagaba sobre conductas deshonestas sólo en exámenes finales, se le agregaron preguntas abiertas previas a las preguntas cerradas que luego fueron codificadas y analizadas mediante la técnica de análisis de contenido. Se solicitaba a los estudiantes ejemplos sobre conducta deshonesta en exámenes, en la presentación de algún trabajo práctico escrito; de conducta deshonesta que afecte a otro estudiante e indagaba acerca de los motivos que llevaban a conductas deshonestas (Espinosa, Castellarin y Biagioni, 2013; Peralta, Ventura, Borgobello y Espinosa, 2014).

Este cuestionario tenía como particularidad que preguntaba al estudiante la frecuencia en la que había tenido alguna conducta académica deshonesta él mismo en exámenes, o si había observado esa misma conducta en sus compañeros. Los resultados de los cuestionarios mostraron, por ejemplo, que el $80 \%$ de los estudiantes respondió que nunca le copió a un compañero durante un examen; sin embargo, el 78\% expresó que en alguna oportunidad vio que sus compañeros se copiaban. Una situación similar sucedió con la copia específica de "machetes" (o "chuletas", piezas de papel en las que los estudiantes tienen anotaciones de las que copian durante los exámenes escritos): mientras que la mayoría declara no haber utilizado esta práctica, al mismo tiempo, expresan haberla visto con alta frecuencia en sus compañeros. Otro resultado para destacar es que más de la mitad de los estudiantes (53\%) declaró que en una o dos oportunidades permitió 
que sus compañeros copien de su examen. Resulta pertinente destacar que es un porcentaje muy elevado en comparación a las restantes preguntas referidas a la conducta propia, resultando que el acto responder el cuestionario parecería ser una conducta deshonesta más.

Los estudiantes avanzados a los que se les solicitó que opinaran acerca de los resultados obtenidos en los cuestionarios, concluyeron que existe una tendencia a ocultar esta conducta en uno mismo y mostrarla como conducta ajena:

Creo que el resultado muestra que, en general, se intenta ocultar la conducta incorrecta (copiar, usar machete) cuando es uno mismo quien la está llevando a cabo, mientras que no hay problemas en remarcarlo cuando es otro quien la realiza [sic].

En relación a la pregunta sobre de qué depende que alguien copie o no, suele atribuirse a una decisión personal basada en la responsabilidad y la madurez de cada uno. Sin embargo, algunos afirmaron que se relaciona con la asignatura en cuanto al control del docente y lo interesante que pudiesen resultar las temáticas desarrolladas.

Este cuestionario tuvo como ventaja un diseño original en el que se contemplaba indagar conductas propias y observación de conductas en compañeros (Sureda et al., 2009) al que se adicionaron preguntas abiertas que permitían analizar el contexto en el que se producían las mismas, intentando bajar los niveles persecutorios que el tipo de indagación podía suscitar (Espinosa et al., 2013; Peralta et al., 2014). Otra ventaja a nivel metodológico fue haber podido entablar un puente con otros científicos y otros contextos a partir de adaptar un instrumento existente, agregando aspectos y formas de indagación propias del equipo local a través de una mirada cualitativa y crítica.

La mayor limitación del instrumento radicó en que el tipo de preguntas fue insuficiente para pensar el contexto de las respuestas, es decir, dieron escasa información sobre esas prácticas en el escenario social de indagación. Esta limitación fue advertida por el equipo de investigación una vez que se habían aplicado numerosos cuestionarios. Por esa razón, y desde la flexibilidad propia de una lógica de investigación cualitativa, se generaron otras formas de indagación que mostraran más cabalmente el objetivo de comprender las prácticas académicas deshonestas en el contexto específico como la indagación a través de correo electrónico.

A pesar de que el equipo de investigación estaba formado por docentes y estudiantes de la Facultad en la que se llevó a cabo el estudio, hubo un aspecto crítico que es pertinente analizar en un artículo como este. Si bien el tema mismo de indagación generaba ciertas reservas, la mayor dificultad a la tarea investigativa fue dada por el instrumento en sí, ya que los cuestionarios generan rechazo en numerosos estudiantes de la institución caracterizada por una formación predominantemente cualitativa y psicoanalítica. Esto fue manifestado tanto en forma oral durante la aplicación de los mismos como por escrito al reverso del instrumento en el que se sugería a los estudiantes que pusieran comentarios libremente acerca del tema o instrumento de indagación. Estas particularidades del contexto generan dificultades a la tarea de indagación que resulta pertinente debatir al diseñar o elegir estrategias metodológicas de acuerdo a los objetivos de un estudio.

\section{El cuestionario implementado por Internet}

En el marco del Proyecto de investigación en curso radicado en el Instituto Rosario de Investigaciones en Ciencias de la Educación (IRICE-CONICET/UNR), titulado "Enseñanza y afiliación en los inicios de la vida universitaria. Una investigación centrada en profesores de primer año" (2015-2017), se implementó una encuesta en línea a docentes de primer año de diferentes Facultades de la UNR. El objetivo del proyecto es indagar la perspectiva de los profesores de primer año sobre diferentes dimensiones implicadas en la enseñanza universitaria: epistemológico-disciplinar, pedagógico-didáctica, ética y política. Así también interesa relevar sus visiones respecto de los estudiantes en su condición de ingresantes a la universidad. Se 
trata de una investigación de corte cualitativo que apunta a recuperar los itinerarios biográficos, trayectorias y experiencias formativas, académicas y profesionales de los profesores de primer año.

Inicialmente, el planteo del proyecto no contemplaba la utilización de cuestionarios. Desde un punto de vista metodológico, se adoptó la Teoría Fundamentada (Grounded Theory) de Glaser y Strauss (1967), en la medida en que el proceso de conceptualización procede de los datos obtenidos en la investigación. Como técnica de recolección de datos se priorizó la entrevista narrativa. Esta consiste en la reconstrucción realizada por los entrevistados de una parte de sus vidas (Bertaux, 2005). El trabajo de campo fue realizado en la Universidad Nacional de Rosario. Se seleccionaron tres Facultades de dicha Universidad: Ciencia Política y Relaciones internacionales; Ciencias Exactas, Ingeniería y Agrimensura y Ciencias Económicas y Estadística, con el propósito de abarcar diversas áreas del conocimiento y estructuras organizacionales. Hasta el momento se realizaron 25 (veinticinco) entrevistas a profesores, decanos, secretarios académicos y estudiantiles, a partir de una muestra heterogénea en relación con variables como la edad, pertenencia institucional y disciplinar, tipo de cargos y dedicaciones, antigüedad en la docencia y en la investigación, tipo de formación, etc.

Una vez finalizado el proceso de realización y análisis de las entrevistas, se consideró que la incorporación de encuestas podría ser un instrumento complementario a los datos ya obtenidos. En efecto, las entrevistas aportaron valiosos insumos desde el punto de vista de la obtención de narraciones tendientes a dar cuenta de las experiencias formativas y profesionales de docentes en ejercicio, insustituibles por un cuestionario. Sin embargo, el carácter reducido de la muestra no favorecía elaborar comparaciones suficientes al interior del cuerpo docente. Más allá de este interés científico, uno de los factores movilizadores principales del diseño del cuestionario fue la dificultad para obtener datos oficiales que permitieran describir los perfiles docentes de las distintas Facultades, de modo tal de poder aproximarse a la pregunta ¿quiénes son los profesores de primer año de la universidad estudiada?

A partir de la decisión tomada, se elaboró un cuestionario a ser autoadministrado por Internet, teniendo en cuenta las principales ventajas de esta modalidad: la posibilidad de acceder a un amplio número de personas con gran rapidez.

El cuestionario estuvo compuesto de 23 preguntas cerradas y una última pregunta abierta destinada a realizar algún comentario o aclaración sobre las respuestas anteriores, o alguna reflexión sobre las problemáticas, desafíos, cuestiones pendientes, etc., que encuentre en su trabajo como docente de primer año.

En función de la carencia de datos oficiales antes mencionada, las primeras preguntas apuntaban a relevar datos sociodemográficos y de hábitos de los docentes como el género, la franja etaria, la antigüedad y tipo de cargo, dedicación, titulaciones, inserción o no en el sistema científico, cantidad estimada de estudiantes a cargo, carreras o cursos de formación docente realizados, modalidad de las clases dictadas, soportes y materiales utilizados.

El resto de las preguntas intentaba, por un lado, obtener información sobre sus percepciones en relación con aspectos estructurales del oficio docente en el primer año de la universidad. Es así que se indagó el grado de acuerdo con las condiciones en que desarrollaban su trabajo, teniendo en cuenta aspectos como relación tiempo y tipo de tarea-salario, condiciones edilicias, distribución del trabajo al interior de las cátedras, equipamiento y recursos disponibles. Por otro lado, se relevaron las posiciones en relación con algunas cuestiones conflictivas en la comunidad universitaria por su trascendencia en términos políticos y sociales. Uno de estos temas era el tipo de sistema de admisión al cual adhieren. Al respecto cabe señalar que la universidad argentina, desde mediados del siglo XX, se inscribe en la tradición del ingreso irrestricto o libre admisión, que sostiene como único requisito para comenzar a cursar una carrera universitaria haber finalizado el nivel anterior (escuela media). Esta modalidad -generadora de procesos de movilidad y democratización social- ha sido interrumpida durante los períodos dictatoriales y reinstalada en los períodos democráticos. Sin embargo, en el presente este punto es motivo de debates y controversias; razón por la cual la elaboración de la pregunta generó dificultades e inquietudes. La preocupación principal radicaba en que si se realizaban preguntas muy directas se corría el riesgo de que los respondentes contesten de modos socialmente aceptados 
dentro de la comunidad universitaria. Por el contrario, si se las elaboraba de forma indirecta podían resultar incomprensibles. Esta disyuntiva se resolvió recurriendo a categorizaciones existentes en la bibliografía disponible sobre el tema. Como puede observarse en el ejemplo que sigue, se presentaron los modos de admisión actualmente existentes solicitando que seleccionen aquel que consideraran más adecuado.

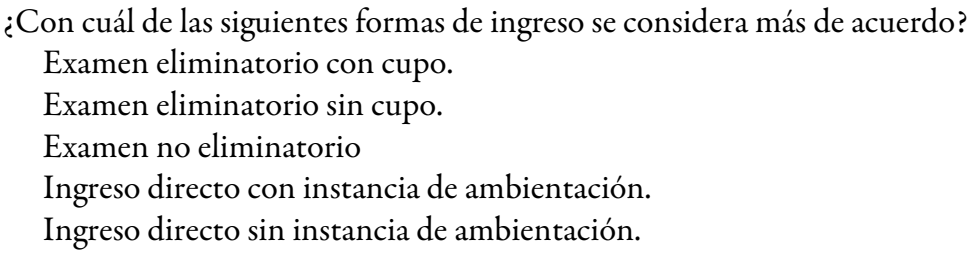

Es preciso advertir sobre una de las limitaciones encontradas en este punto. Al analizar las respuestas, se observó que la mayoría (47\%) se consideraba de acuerdo con la opción Ingreso directo con instancia de ambientación. Sin embargo, en la última pregunta, que solicitaba realizar comentarios u observaciones sobre los puntos anteriores, una respondente expresó "no saber bien" qué significaba "instancia de ambientación". Lo anterior condujo a pensar en las limitaciones de dar por supuestas categorías recuperadas de fuentes teóricas o utilizadas con asiduidad por los investigadores. Una mayor explicitación o la utilización de un lenguaje de uso masivo, podría haber evitado dudas innecesarias. A su vez, este caso constituye un claro ejemplo de uno de los inconvenientes más comunes de los cuestionarios por Internet, que consiste en la ausencia de una persona que responda a las dudas originadas en la lectura del cuestionario (Díaz, 2012).

Otra de las cuestiones que se pretendía indagar era el modo en que los docentes de primer año explicaban las altas tasas de abandono en el ciclo inicial, que en las universidades argentinas ronda el $40 \%$. Interesaba analizar en qué medida las causas de este fenómeno eran atribuidas a dificultades de los propios estudiantes o a responsabilidades institucionales y pedagógicas. En este caso, también se recuperó literatura específica sobre el tema, para considerar diferentes perspectivas aportadas por investigaciones teóricas y empíricas. Se decidió incorporar una escala de alternativas múltiples de tipo ordinal (en este caso las opciones eran nada, poco, algo, bastante, mucho) para observar el peso de los distintos factores mencionados en dichas investigaciones en las percepciones de los docentes:

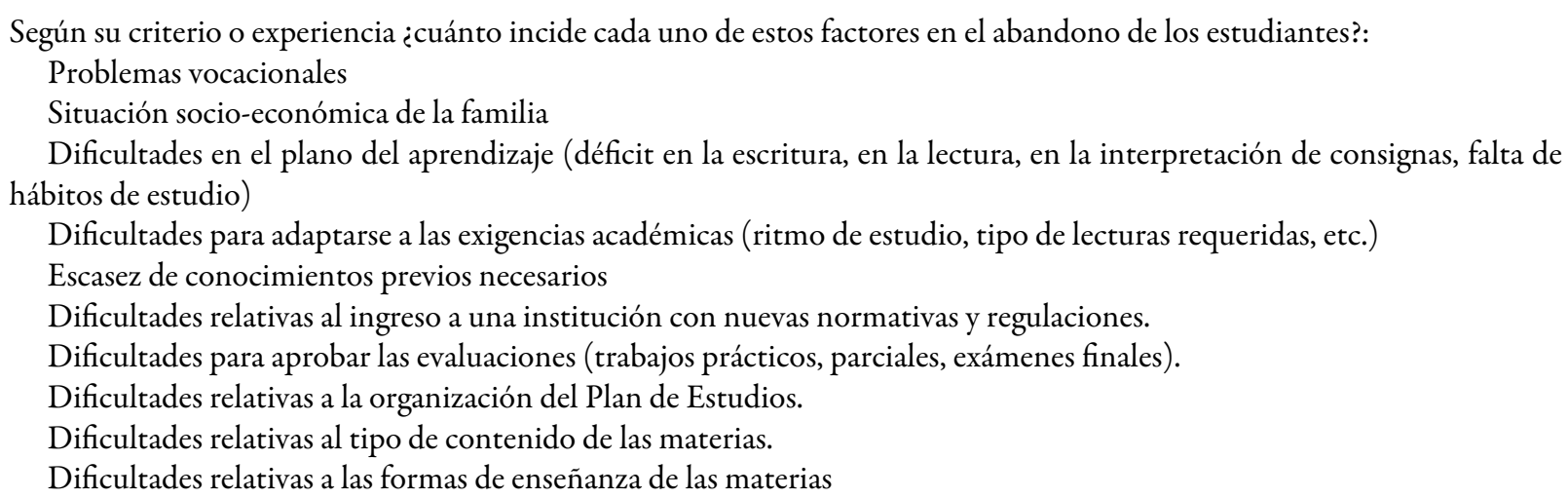

Sobre este tipo de preguntas, que incluyen múltiples variables y en las que se solicita una valoración por cada una de estas, cabe advertir la necesidad de realizar una lectura cruzada de las respuestas al interior de un mismo respondente (mirada horizontal) en el momento del análisis de las respuestas. En este sentido, mientras que una entrevista proporcionaría la posibilidad de percibir holísticamente la perspectiva del entrevistado, el cuestionario resigna la singularidad de los respondentes en miras a la sumatoria de las respuestas obtenidas (a menos que se recurra a técnicas estadísticas específicas que recuperen los perfiles individuales de los respondentes). 


\section{El cuestionario en un estudio cuasiexperimental}

Este estudio formó parte de un proyecto más amplio, la Tesis Doctoral de una de las autoras, radicado en el Laboratorio de Psicología Social Experimental del Instituto Rosario de Investigaciones en Ciencias de la Educación. En el proyecto en su conjunto se analizaron las funciones tutoriales en la universidad de las personas que se inician en la docencia universitaria. En el estudio que aquí se reporta se realizó un testeo cuasiexperimental de los roles docentes en una situación controlada (Borgobello, 2010; Borgobello, Peralta y Roselli, 2013).

El experimento fue replicado en dos casos: una cátedra de ciencias humanas y otra de ciencias exactas. En cada caso, los docentes debían enseñar un tema a grupos de diez estudiantes divididos al azar. Participaron como enseñantes en cada situación experimental un docente con amplia experiencia (habituado a dictar clases teóricas), un auxiliar de primera (graduado reciente habituado a trabajar con grupos reducidos de estudiantes en clases prácticas) y un auxiliar de segunda (estudiante de la carrera cuyo rol habitual en la cátedra es de acompañamiento y colaboración con el docente de trabajos prácticos, ya que por reglamento no puede estar a cargo de clase alguna).

Se les explicó a los docentes voluntarios el objetivo general del estudio y, una semana antes del experimento, se les dio un texto fuente del que se tomaron las preguntas del cuestionario postest. Las clases fueron grabadas en audio y desgrabadas. Las tres clases trascriptas de cada caso fueron evaluadas por cinco jueces expertos externos que desconocían la naturaleza del experimento, es decir, no sabían que los docentes que evaluaban tenían distintos grados de experticia.

Este estudio se enfocó desde la psicología sociocultural que postula que es posible pensar que los procesos de enseñanza y aprendizaje están configurados en un espacio social que les da la forma particular. Desde González-Rey (2008), serían formas de acción incrustadas en la trama vital y en la vida social de las personas que se plasman, por ejemplo, en las prácticas docentes concretas.

En este sentido, el objetivo de estas situaciones experimentales fue analizar las acciones plasmadas en las prácticas docentes áulicas en contextos similares que no pueden ser observadas en situaciones naturales porque, por ejemplo, los auxiliares de segunda no dan clases frente a curso. Con esta meta, en el diseño se mantuvieron constantes la cantidad de estudiantes de cada grupo y el texto fuente de cada caso. Adicionalmente, se buscó comparar el aprendizaje de los tres grupos de estudiantes y las evaluaciones que ellos hicieron de las clases a las que asistieron. En el experimento en su conjunto se analizaron, además de los cuestionarios, las características de la interacción producidas en cada clase a partir de la observación y las desgrabaciones.

Se diseñaron cuatro cuestionarios en formato papel. Para evaluar el conocimiento previo y el conocimiento adquirido de los estudiantes, se utilizó un pretest general con preguntas abiertas sobre los temas trabajados en las clases y postest específico basado en el texto fuente utilizado en cada caso. La evaluación de las clases por parte de los jueces se llevó a cabo a través de un cuestionario que permitía puntuar en las tres clases organización conceptual y del tiempo, claridad y profundidad conceptual. Finalmente, luego del postest se aplicó a los estudiantes un cuestionario de opinión y evaluación de la clase en el que se preguntaba por el grado de complejidad del tema enseñado, se pedía una puntuación de la clase y se indagaba qué porcentaje del aprendizaje consideraban que habían logrado.

La mayor ventaja de la utilización de cuestionarios en este tipo de diseños es que permiten comparar, objetivo central del diseño pretest-postest. De algún modo, estos diseños se encuentran destinados a comparar lo incomparable en escenarios naturales, a hacer posible poner en un plano de igualdad a personas que, en casos como este, desarrollan actividades docentes distintas.

Los resultados de los cuestionarios que completaron los jueces externos y otros análisis realizados (Borgobello, 2010) en las situaciones de dictado de clases muestran características de los roles habituales en los tipos de clases dadas habitualmente por los docentes. Por ejemplo, las clases a cargo de los titulares de 
cátedra fueron las más expositivas a pesar de estar trabajando solo con diez estudiantes, mientras que las clases de los auxiliares de primera fueron más participativas.

Este tipo de diseño cuasiexperimental permite reflexionar sobre la cristalización de los roles en la práctica docente, al tiempo que demuestra similitudes entre casos y formas de acción consolidadas más allá de los contextos específicos en los que se desarrollan dichas prácticas docentes.

Una de las dificultades halladas, inicialmente atribuidas al diseño, fue que los estudiantes que tomaron clases con los auxiliares obtuvieron mejores puntajes en los postest que los que lo hicieron con los expertos. Esta característica estaría asociada directamente a la sujeción al texto fuente demostrada por los auxiliares y que los postests estaban estructurados de acuerdo a esa fuente. Cabe destacar que en las clases reales sucede algo similar: los conceptos habitualmente evaluados se suelen encontrar en los textos. Estos resultados lejos están de demostrar que los expertos no tendrían relevancia en el dictado de clases ya que son ellos los que permiten situar a los textos en contextos más amplios. Por el contrario, mostrarían la importancia de la diversidad de roles y del trabajo en equipo de las cátedras universitarias.

Una de las discusiones teóricas de mayor relevancia dadas en el marco de este estudio fue la medición de aprendizaje a través de cuestionarios. Los postest tomados luego de las clases no medirían aprendizaje propiamente dicho sino el recuerdo inmediato. Es decir, el concepto de aprendizaje, al menos desde posturas constructivistas, tiene una complejidad tal que no es posible asimilarlo a recuerdo y, mucho menos, a recuerdo inmediato posterior (Borgobello, 2010).

En cuanto a la construcción de los instrumentos, el que generó las mayores dificultades fue el cuestionario destinado a la evaluación comparativa de las clases por parte de los jueces externos voluntarios. Era necesario, en el marco de los objetivos del experimento, testear con el mismo cuestionario todas las clases, y sin embargo, contábamos con expertos en dos áreas del conocimiento altamente disímiles, participantes de culturas académico-institucionales distintas. El cuestionario debía ser un instrumento único para ambos casos y simple de comprender. Esto requirió varias pruebas piloto para ajustar el lenguaje tanto para científicos de ciencias exactas como de ciencias sociales. Finalmente, se dejó espacio para que los jueces justificasen sus decisiones y agregasen comentarios si lo consideraban necesario, dando así mayor libertad a los respondentes y aportando datos cualitativos útiles para comprender las respuestas comparativamente.

Una dificultad adicional del trabajo con cuestionarios en este estudio fue que los estudiantes de ciencias humanas no se encontraban acostumbrados a responder cuestionarios cerrados como evaluación de conocimiento. Si bien el grupo de voluntarios mostró amplia colaboración con el trabajo de investigación, el tipo de instrumento generó ciertas resistencias.

\section{DisCuSión Y CONCLUSIONES}

Este artículo se propuso poner en debate a través de ejemplos concretos las ventajas y limitaciones del uso de cuestionarios para investigar en la universidad. Con este objetivo, señalamos la ambigüedad terminológica (Archenti, 2007; Canales et al., 1994; Hernández-Sampieri et al., 2008) y metodológica implícita que rodea a los términos encuesta y cuestionario: einstrumentos, técnicas, estrategia, métodos, diseños, tipos de investigación? Si bien existen acuerdos generales sobre ventajas y limitaciones (Canales et al., 1994; Coomber, 1997; Díaz, 2012; Hernández-Sampieri et al., 2008), la cuestión teórico-metodológica de fondo parece seguir siendo un tema insuficientemente problematizado. Por esta razón, aquí se examinaron cuatro estudios en los que se utilizaron cuestionarios, entendidos como instrumentos o técnicas en el marco de la encuesta como método. Las limitaciones encontradas en cada caso mostraron la necesidad de concebirlos en enfoques complejos que los superen como abordaje único, especialmente en proyectos interesados por lo singular. Asimismo, el objetivo de utilizar casos radicó en contextualizar la discusión en experiencias de investigaciones ya implementadas. Las situaciones paradigmáticas presentadas fueron: 
- estudio internacional aplicado en una cobertura temporal, geográfica y lingüística extensa en el que se analizó la satisfacción de los participantes respecto de la formación recibida;

- estudio sobre temas sensibles para los respondentes; en este caso, la deshonestidad académica en estudiantes universitarios;

- una consulta en línea destinada a docentes universitarios de primer año orientada a indagar cuestiones relativas a su desempeño laboral.

- estudio cuasiexperimental destinado a analizar las prácticas docentes en roles distintos dentro del organigrama de la cátedra universitaria.

La selección de estudios para el análisis buscó reunir diversos equipos de investigación, temas disímiles dentro del ámbito universitario y procedimientos investigativos variados. En cada estudio, los respondentes de los cuestionarios presentaron diferentes perfiles: profesores en situación de capacitación, docentes universitarios de primer año, estudiantes de grado en distintos momentos de la carrera y evaluadores expertos. Si bien la característica más sobresaliente de la selección de estudios fue la diversidad, estos tuvieron dos puntos en común. El primero atañe al enfoque complejo (Achilli, 2005) en equipos de investigación caracterizados por formación en el análisis cualitativo de datos. El segundo punto de coincidencia -y no ajeno a la orientación teórico-metodológica mencionada- es que todos los cuestionarios fueron diseñados ad hoc, es decir, no fueron estandarizados en un sentido clásico.

La presentación se estructuró a partir de seis ejes de análisis: contexto de radicación y realización de los estudios, enfoque teórico-metodológico, objetivos de los instrumentos en el marco de cada estudio, características del soporte, ventajas, limitaciones y dificultades encontradas en la tarea investigativa.

Los contextos institucionales de los proyectos de investigación que enmarcaron el uso de los instrumentos que se analizaron fueron, como se dijo, un organismo internacional que involucra universidades nacionales de seis países, una Facultad en particular y un organismo nacional de investigación.

El enfoque teórico-metodológico de abordaje de los proyectos tuvo como característica común la lógica de construcción complejo-dialéctica (Achilli, 2005), más allá de las claras divergencias que pueden advertirse entre los proyectos de investigación marco.

Con respecto a los objetivos de los proyectos e instrumentos de cada uno, el primer estudio presentado estuvo destinado a ampliar el área de uso de un recurso educativo, mientras que el cuestionario se propuso específicamente relevar el grado de satisfacción respecto del recurso en sí y de las capacitaciones proporcionadas.

En el estudio abocado a conocer y analizar contextualmente las opiniones de los estudiantes sobre prácticas transgresoras en las que incurrían, el cuestionario aplicado se enfocó en la recolección de ejemplos que daban los estudiantes sobre conductas deshonestas en circunstancias variadas como escritura académica y trabajos grupales, y luego se indagaba frecuencia y tipo de conducta deshonesta en exámenes escritos.

En el proyecto de uso en línea del cuestionario centrado en indagar las perspectivas de los profesores de primer año sobre las dimensiones implicadas en la enseñanza universitaria, se buscó conocer las condiciones de trabajo, sus percepciones sobre la enseñanza en los primeros ciclos de la universidad y sus visiones sobre los estudiantes en su condición de ingresantes, considerando las dificultades y desafíos propios de ese momento de transición.

Los cuestionarios que se utilizaron en el último estudio expuesto provenían de un proyecto dedicado a analizar las funciones tutoriales de los auxiliares a través de un enfoque multimétodo (estudio de fuentes documentales, etnográfico y experimental). El objetivo del estudio era comparar la enseñanza de tres docentes en roles y disciplinas distintas (diseño 3x2). Uno de los instrumentos apuntaba a la evaluación de conocimientos previos (pretest) de los estudiantes, otro a la evaluación de las clases por parte de expertos en las temáticas abordadas en las clases, el tercero destinado a medir el aprendizaje (postest) y, el último, para evaluar las clases desde la perspectiva de los estudiantes. 
En cuanto al soporte, se recurrió tanto al papel en forma exclusiva como únicamente a internet y a la combinación de ambos (tradicional y en línea con acceso restringido).

Con respecto a las ventajas proporcionadas por el tipo de instrumento, el cuestionario como técnica permitió poner en plano de igualdad para medir y comparar, por ejemplo, al evaluar implementaciones en contextos, instituciones, momentos y objetos en las capacitaciones o al diseñar una situación experimental con los controles del caso. Otra ventaja técnica visible en casi todos los casos abordados fue la posibilidad de expresar matices respecto al grado de conformidad restringiendo, a la vez, los grados de valoración. Particularmente en el estudio sobre deshonestidad académica, el diseño original del instrumento permitió indagar conductas propias y de compañeros que suelen ocultarse.

Al trabajar en proyectos con enfoque complejo, el uso de preguntas abiertas dio lugar a recabar argumentaciones y a incorporar aspectos cualitativos y contextuales en los análisis. La garantía de anonimato (reforzada por la masividad y el tipo de soporte, según los casos) permitió la expresión más liberada en temas delicados por aspectos morales o de lo esperable como políticamente correcto en determinados grupos sociales.

En el caso específico del cuestionario aplicado completamente en línea, este permitió acceder a docentes a través de intermediarios institucionales a los que no se hubiera accedido de otro modo. Es decir, con el envío de un solo correo electrónico se accedió al mismo tiempo al conjunto de docentes desde una fuente para ellos confiable y, adicionalmente, la recolección de las respuestas no requirió intermediarios.

En cuanto a los aspectos negativos se decidió desagregarlos en limitaciones y dificultades. Las primeras refieren a la etapa del diseño, mientras que las dificultades, a la implementación de los cuestionarios. En la fase del diseño, en algunos de los estudios se observó la necesidad de atender al ajuste entre las preguntas y las opciones proporcionadas en las respuestas, mientras que en otros (concretamente el de temas sensibles), el tipo de preguntas fue insuficiente para pensar el contexto de las prácticas indagadas en el escenario social de investigación. Esta limitación se solucionó a través de otras vías de indagación predominantemente cualitativas a través del uso de correo electrónico.

También en la fase de diseño y en el caso específico del estudio internacional, se advirtió la importancia de preservar los matices semánticos en las traducciones del protocolo. Otra de las limitaciones encontradas en la comprensión de las preguntas fue haber dado por supuestas categorías recuperadas de fuentes teóricas o utilizadas con asiduidad por los investigadores. Esta cuestión podría superarse en futuras aplicaciones recurriendo a un lenguaje de uso más general o ampliando las explicaciones en las propias preguntas. Finalmente, otra limitación fue tener que resignar la singularidad de los respondentes en miras a la sumatoria de las respuestas obtenidas.

Con respecto a las dificultades, propias de la etapa de implementación, los diversos estudios coinciden en que no siempre se logró cooperación suficiente para responder, especialmente las preguntas abiertas. En el caso particular del estudio sobre temas sensibles, el tipo de instrumento generó cierto rechazo en una institución caracterizada por una formación predominantemente cualitativa y psicoanalítica. Una dificultad adicional hallada en la implementación se centró en el escaso hábito de los estudiantes de ciencias humanas para contestar cuestionarios cerrados, algo que se repitió en diferentes casos como en la evaluación de conocimiento del diseño cuasiexperimental y en el estudio sobre deshonestidad. En el estudio experimental, se dio el obstáculo de que un mismo cuestionario debía ser evaluado por expertos en dos áreas del conocimiento altamente disímiles, por lo que se dejaron espacios abiertos para aclaraciones.

Una conclusión que se desprende de los estudios presentados es la necesidad de leer los resultados en contexto. Un ejemplo claro de ello es el estudio destinado a comparar roles docentes en el que se halló que los estudiantes "aprendieron más" en las clases de los auxiliares. Sin embargo, los docentes con más experiencia enmarcaron e historizaron el contenido trabajado en clases dando la posibilidad a los estudiantes de aprendizajes que superaran el recuerdo inmediato posterior. En líneas generales se percibió 
cierta insuficiencia en la riqueza de los datos para producir interpretaciones más elaboradas en base a las respuestas de los informantes.

Más allá del momento en que se aplique, la proyección temporal es un requerimiento propio del cuestionario en enfoques flexibles como los aquí presentados: anticipar las posibles respuestas, y aún el devenir de la investigación cuando la implementación es a largo plazo.

Asimismo, las críticas a los cuestionarios suelen referir más que a la naturaleza del instrumento en sí, a su confección y/o uso limitado o desprolijo. Así, se confunden las desventajas técnicas con descuidos de quien las diseña y/o implementa.

Una reflexión adicional es que suele concebirse al cuestionario como instrumento que eludiría el sesgo del investigador dada su protocolización habitual. Sin embargo, se omite de manera generalizada el sesgo producido por quienes lo elaboran como si el producto estuviera libre de valoraciones. Las preguntas acerca de las percepciones, representaciones y creencias ponen en evidencia la naturaleza subjetiva de las respuestas que suelen quedar dejadas de lado en algunas lecturas que asocian las respuestas con la realidad a secas. Esta ilusión de transparencia puede tener distintos orígenes que van desde la ingenuidad hasta la intención de manipular datos, reforzando argumentos a través de la legitimidad que brindan los estudios empíricos. Otros ejemplos sobre el tema que debieran ser objeto de futuras investigaciones podrían ser, entre otros: la presión experimentada por los respondentes ante la presencia del investigador, la situación en la cual las respuestas a un cuestionario pueden tener consecuencias en la estabilidad laboral o el desempeño académico y la solidaridad o identificación entre actores.

\section{REFERENCIAS}

Achilli, E. (2005). Investigar en antropología social. Los desafios de transmitir un oficio. Rosario: Laborde.

Acosta, B. (2012). Evaluación de las propiedades psicométricas del cuestionario de evaluación docente (DOCENTIA) para su uso en una muestra mexicana. Revista Iberoamericana de Evaluación Educativa, 5(2), 182-197.

Archenti, N. (2007). El sondeo. En A. Marradi, N. Archenti y J.I. Piovani (Eds.), Metodología de la Ciencias Sociales (pp. 203-2014). Buenos Aires: Emecé.

Becher, T. (2001). Tribus y territorios académicos. La indagación intelectual y las culturas de las disciplinas. Barcelona: Gedisa.

Bertaux, D. (2005). Los relatos de vida. Perspectiva etnosociológica. Barcelona: Bellaterra.

Borgobello, A. (2010). Las mediaciones tutoriales en la enseñanza universitaria: el rol de los auxiliares de primera y de segunda en la UNR (Tesis Doctoral), Universidad Nacional de San Luis, Argentina.

Borgobello, A., Peralta, N. y Roselli, N. (2013). Interaction among experience, teaching performance and student's learning at university level. Estudos de Psicologia, 30(1), 169-176.

Bustamante, E., Bustamante, F., González, G. y Bustamante, L. (2016). El burnout en la profesión docente: un estudio en la escuela de bioanálisis de la Universidad de Carabobo Sede Aragua, Venezuela. Medicina y seguridad del trabajo, 62(243), 111-121.

Cala Aiello, R. y Riera García, M. (2014). Determinación de los estilos de aprendizaje de estudiantes de 1er curso de ing. industrial y electrónica de la Universidad Técnica Del Norte. Ibarra. Ecuador. Revista de Estilos de Aprendizaje, $7(14), 43-67$.

Canales, F., Alvarado, E., y Pineda, E. (1994). Metodología de la investigación. Manual para el desarrollo de personal de salud. Washington: OPS.

Coomber, R. (1997). Using the Internet for Survey Research. Sociological Research Online, 2(2), 1-18.

De-Sena, A., y Lisdero, P. (2015). Etnografía virtual: aportes para su discusión y diseño. En A. De-Sena, Caminos cualitativos (pp. 71-100). Buenos Aires: Ciccus.

Díaz, V. (2012). Ventajas e inconvenientes de la encuesta por Internet. Papers, 97(1), 193-223. 
Espinosa, A., Castellarin, M. y Biagioni, F. (2013). Prácticas académicas deshonestas en exámenes escritos. Análisis de una muestra de alumnos de primer año de la Carrera de Psicología de la UNR. Ponencia presentada en V Congreso Internacional de Investigación y Práctica Profesional en Psicología, Universidad de Buenos Aires. Recuperado de: https://www.aacademica.org/000-054/427.pdf

Falicoff, C.B., Odetti, H.S. y Domínguez Castiñeiras, J.M. (2014). Competencia científica de estudiantes que ingresan y egresan de la Universidad. Enseñanza de las ciencias, 32(3), 133-154.

Glaser, B. y Strauss, A. (1967). The discovery of grounded theory: strategies for qualitative research. New York: Aldine.

González-Rey, F. (2008). Subjetividad social, sujeto y representaciones sociales. Diversitas: Perspectivas en Psicologia, 4(2), 225-243.

Hernández-Sampieri, R., Fernández-Collado, C., y Baptista-Lucio, M.P. (2008). Metodología de la investigación. México: McGrawHill.

Manchini, N., Penhos, M. y Suárez, O. (2016). El impacto de la Educación en Derechos Humanos en la Universidad Argentina del siglo XXI. Bauru, 4(1), 33-61.

Medrano, L.A., Moretti, L., Ortiz, A. y Pereno, G. (2013). Validación del Cuestionario de Regulación Emocional Cognitiva en Universitarios de Córdoba, Argentina. Psykhe, 22(1), 83-96.

Noriega Biggio, M., Vázquez, S.M. y García, S.M. (2015). Deserción en estudiantes de nuevo ingreso a carreras de diseño. El caso de la Universidad de Buenos Aires, Argentina. Revista Actualidades Investigativas en Educación, 15(1), 1-23.

Peralta, N., Ventura, A.C., Borgobello, A. y Espinosa, A. (2014). Análisis descriptivo de las posibles prácticas académicas deshonestas de los estudiantes de sexto año de psicología según sus propias opiniones. En C. López; L. Bulacio y M. Migliaro (Coord.), Divulgación de la producción científica y tecnológica de la UNR (pp. 643-646). Rosario: UNR Editora.

Pérez, C., Vaccarezza, G., Aguilar, C., Coloma, K, Salgado, H., Baquedano, M., Chavarría, C. y Bastías, N. (2016). Cuestionario de prácticas pedagógicas: análisis de su estructura factorial y consistencia interna en docentes de carreras de la salud. RevMedChile, 144, 795-805.

Schmelkes, S. (2001). La combinación de estrategias cuantitativas y cualitativas en la investigación educativa: Reflexiones a partir de tres estudios. Revista Electrónica de Investigación Educativa, 3(2), 82-94. Recuperado de http://www.redalyc.org/pdf/155/15503205.pdf

Sureda-Negre, J., Comas-Forgas, R., y Gili-Planas, M. (2009). Prácticas académicas deshonestas en el desarrollo de exámenes entre el alumnado universitario español. Estudios sobre Educación, 17(1), 103-122.

Ventura, A.C. y Moscoloni, N. (2015). Estilos de enseñanza y aprendizaje en las aulas universitarias: la dimensión cognitiva y social de la estilística. Psicología, Conocimiento y Sociedad, 5(1), 82-109.

\section{Notas}

1 Investigadora categoría independiente del Consejo Nacional de Investigaciones Científicas y Técnicas de Argentina en el Instituto Rosario de Investigaciones en Ciencias de la Educación de Rosario, Argentina. Es Doctora en Humanidades y Artes, mención Ciencias de la Educación por la Universidad Nacional de Rosario, donde se desempeña como Profesora Adjunta de Trabajo de Campo. En la actualidad participa de un proyecto internacional sobre educación en las Ingenierías subsidiado por la Unión Europea.

2 Investigadora categoría asistente del Consejo Nacional de Investigaciones Científicas y Técnicas de Argentina en el Instituto Rosario de Investigaciones en Ciencias de la Educación de la ciudad de Rosario, Argentina. Es Doctora en Psicología por la Universidad Nacional de San Luis. Se desempeña como Jefe de Trabajos Prácticos de Metodologías de la Investigación en Psicología de la Facultad de Psicología de la Universidad Nacional de Rosario.

3 Investigadora categoría asistente del Consejo Nacional de Investigaciones Científicas y Técnicas de Argentina en el Instituto Rosario de Investigaciones en Ciencias de la Educación de la ciudad de Rosario, Argentina. Es Doctora en Ciencias Sociales por la Universidad de Buenos Aires. Se desempeña como Jefe de Trabajos Prácticos en el Núcleo Socio Educativo en la Carrera de Ciencias de la Educación de la Facultad de Humanidades y Artes de la Universidad Nacional de Rosario. 